\title{
Exercise interveNtion outdoor proJect in the cOmmunitY for older people - results from the ENJOY Seniors Exercise Park project translation research in the community
}

Pazit Levinger ${ }^{1,2,3^{*}}$, Maya Panisset ${ }^{1}$, Jeremy Dunn ${ }^{1}$, Terry Haines ${ }^{4}$, Briony Dow ${ }^{1,5,6}$, Frances Batchelor ${ }^{1,6,7}$, Stuart Biddle ${ }^{8}$, Gustavo Duque ${ }^{9}$ and Keith D. Hill ${ }^{3}$

\begin{abstract}
Background: Many research studies evaluate physical activity interventions for older people in the community, however relatively few successfully promote maintenance of physical activity beyond the completion of the intervention. This study aimed to implement and evaluate the effects of sustained engagement in physical activity on mental, social and physical health outcomes through the use of the Seniors Exercise Park physical activity program for older people (the ENJOY project).

Method: People aged $\geq 60$ years underwent a 12-week structured supervised physical activity program using outdoor exercise park equipment followed by 6 months unstructured independent use of the exercise park. Participants were assessed at baseline, 3 months and 9 months and completed a test battery evaluating physical activity, physical function and health related quality of life measures. Repeated measures ANOVA was used to compare differences between baseline, 3 and 9 months.

Results: Of the 95 participants, 80 (84.2\%) completed the 3 months supervised program, and 58 (61\%) completed the 9 month assessment (the latter impacted by COVID-19 restrictions). A significant increase in physical activity level was demonstrated following the 12 weeks intervention $(p<0.01)$. Significant improvements were also demonstrated in all physical function measures $(p<0.01)$, self-rated quality of life $(p<0.05)$, wellbeing $(p<0.01)$, fear of falls $(p<0.01)$, falls risk $(p<0.01)$, depressive symptoms $(p=0.01)$ and loneliness $(p=0.03)$ at 3 months. At the 9 months follow up, significant improvements from baseline were demonstrated in the frequency, duration and total of physical activity level $(p<0.05)$, and all physical function measures $(p<0.05)$, with no decline in these measures from 3 to 9 months. At 9 months, significant changes were observed in the health related quality of life mobility and self care domains with reductions in both fear of falls and falls risk $(p<0.05)$.

(Continued on next page)
\end{abstract}

\footnotetext{
* Correspondence: p.levinger@nari.edu.au

${ }^{1}$ National Ageing Research Institute, Melbourne, Australia

${ }^{2}$ Institute for Health and Sport, Victoria University, Melbourne, Australia

Full list of author information is available at the end of the article
}

(c) The Author(s). 2020 Open Access This article is licensed under a Creative Commons Attribution 4.0 International License, which permits use, sharing, adaptation, distribution and reproduction in any medium or format, as long as you give appropriate credit to the original author(s) and the source, provide a link to the Creative Commons licence, and indicate if changes were made. The images or other third party material in this article are included in the article's Creative Commons licence, unless indicated otherwise in a credit line to the material. If material is not included in the article's Creative Commons licence and your intended use is not permitted by statutory regulation or exceeds the permitted use, you will need to obtain permission directly from the copyright holder. To view a copy of this licence, visit http://creativecommons.org/licenses/by/4.0/ The Creative Commons Public Domain Dedication waiver (http://creativecommons.org/publicdomain/zero/1.0/) applies to the data made available in this article, unless otherwise stated in a credit line to the data. 
(Continued from previous page)

Conclusion: The Seniors Exercise Park may be an effective modality for improving and sustaining older people's physical function and wellbeing and can be an important public health infrastructure investment in promoting physical activity for older people. Future work should focus on wider implementation of the program and on scaling up this initiative to achieve public health benefit for the community.

Trial registration: Trial registration number ACTRN12618001727235, Date of registration 19th October 2018, https://www. anzctr.org.au/Trial/Registration/TrialReview.aspx?id=375979

Keywords: Physical activity, Older people, Falls prevention, Seniors Exercise Park, Outdoor

\section{Background}

The world's population is ageing rapidly, with the number of older people age 65 and over projected to more than double by 2050 [1]. The number of Australians aged 65 and over is expected to increase from around 2.5 million in 2002 to 6.2 million in 2042 [2]. Physical activity is one of the key behavioral factors to positively impact health outcomes, including reduction of risk of chronic diseases, cognitive and functional decline, and improvement in mental health [3]. Increase in physical activity can also minimise the burden on the health care system [4]. Despite the strong evidence around the importance of physical activity, older people do not regularly undertake physical activity [5], with less than $25 \%$ of older Australians meeting the recommended physical activity guidelines [6].

There has been considerable research into physical activity interventions for older people in the community, but interventions that successfully promote maintenance of physical activity beyond the completion of the intervention are limited [7, 8]. Various methodological challenges exist that often limit translation of physical activity programs into practice, these include: lack of evidence of transferability of trial results to the community setting, insufficient local expertise to roll out community exercise programs, and inadequate infrastructure to integrate evidence based programs into community practice [9]. Interventions that are designed to be conducted in a community setting with community engagement have the potential to be sustained beyond the trial period and have shown to be effective in increasing and promoting physical activity $[10,11]$.

In recent years, outdoor environments and associated infrastructure features (e.g., exercise equipment) have been recognized as an important investment to promote regular physical activity [12, 13]. Hence, the design of an age friendly 'active environment' has been recommended as one of the strategies to increase physical activity at a population level [14]. In 2012 we commenced our research work in the area of age friendly active spaces for older people with the utilization of outdoor exercise equipment specifically designed for older people (the Seniors Exercise Park). The Seniors Exercise Park program was designed to actively promote community well-being through the provision of a unique exercise and social support program. In a small 18 week randomized controlled trial (RCT), we demonstrated the effectiveness of the Seniors Exercise Park program on improving physical function and social health in older people $[15,16]$. These preliminary positive outcomes indicated the need for investigation of its sustained impact on physical and social health outcomes, and its potential wider usage in the community on a larger scale with local governments' (councils') engagement. Therefore, the aim of the present study was to implement and evaluate the effects of sustained engagement in physical activity on physical, mental, social and health outcomes through the use of the Seniors Exercise Park physical activity program for older people (the ENJOY project).

\section{Methods}

\section{Design and setting}

This study was a multi-site prospective study with a pre and post intervention design and 9 month follow up. Participants underwent a 12-week structured supervised physical activity program using outdoor exercise park equipment followed by a 6 month unstructured physical activity program, including ongoing unsupervised access to the exercise park. Each exercise session was followed by a social gathering with morning/afternoon tea provided by the research team. Participants were assessed at baseline, post intervention (3 months) and 9 months follow up time points as detailed in Fig. 1. The study was designed according to the Transparent Reporting of Evaluations with Nonrandomized Designs (TREND) [17] which complements the widely adopted Consolidated Standards Of Reporting Trials (CONSORT) statement developed for randomized controlled trials [18]. Ethical approval was obtained from the Melbourne Health Human Research Ethics Committee, Melbourne (Application ID. HREC/18/MH/286, local number 2018.238). All participants provided informed consent. The full description of the study's methods, design, and procedure can be found in the trial protocol [19].

\section{Study population \\ Inclusion criteria}

Older people were eligible to participate in the study if they: 


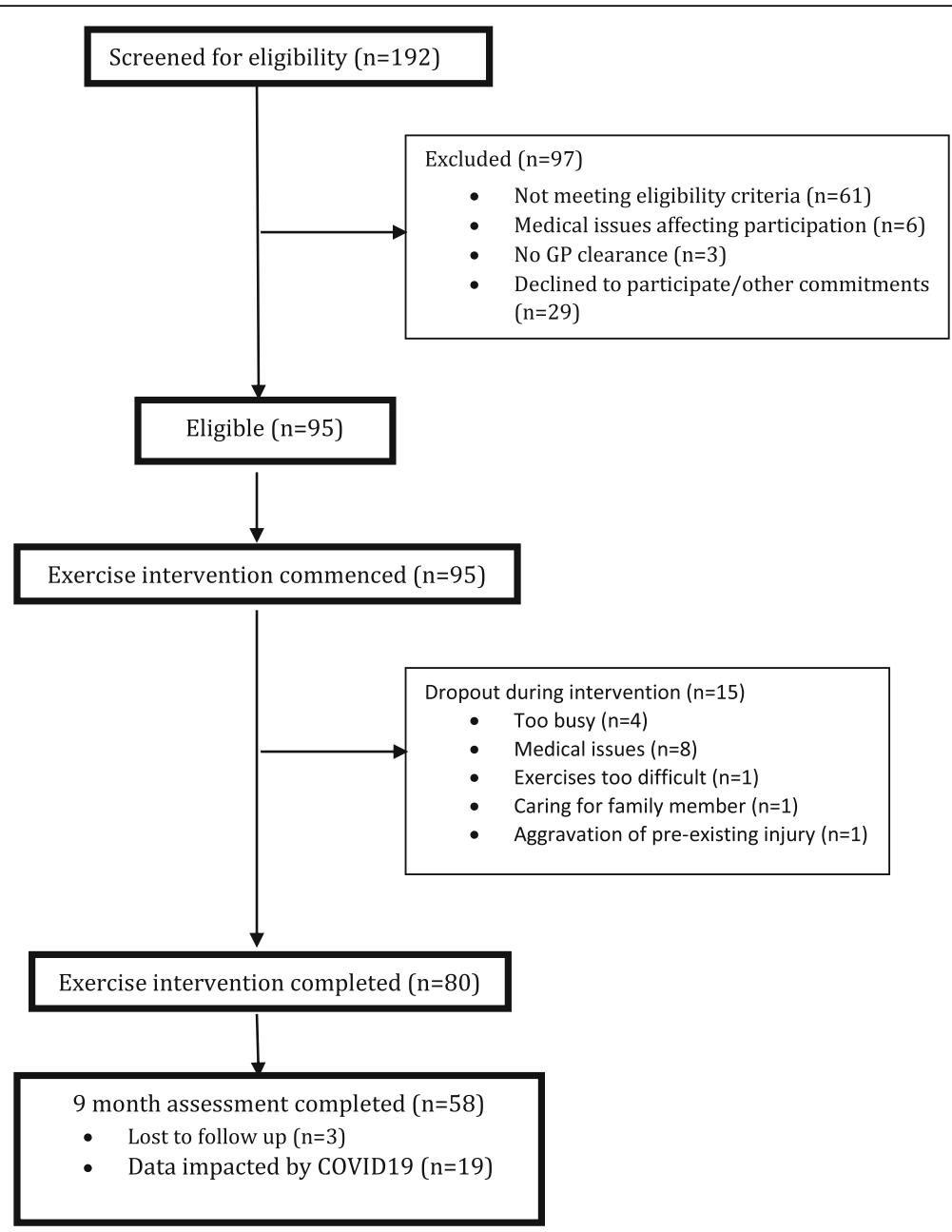

Fig. 1 Flow diagram of recruitment and drop out

1) were aged 60 years and over living in the community (i.e. not living in an institution, such as a nursing home); 2) had one or more falls in the previous 12 months or were concerned about having a fall; 3) were generally independent around the house (able to take care of themselves) and in the community (e.g. able to walk away from home to visit local stores, friends, and other local venues), and able to attend the outdoor exercise park; 4) were able to walk outdoors and use the exercise equipment with no more gait aid support than a single point stick 5) did not have cognitive impairment (Abbreviated Mental Test Score (AMTS) > 7/10) [20].

\section{Exclusion criteria}

Older adults were excluded from this study if they: 1) had neurological or musculoskeletal conditions limiting walking to less than one block; 2) had a history of stroke, Parkinson's disease, or other neurological disorder impacting on mobility; 3) were unable to understand conversational
English; 4) were taking part in a structured resistance training and/or an organised balance training program more than once a week; 5) met the Australian physical activity recommendations of $150 \mathrm{~min}$ of physical activity / week [21]; 6) had any documented medical condition or physical impairment that was deemed by their medical practitioner to contraindicate their inclusion.

\section{Recruitment}

Older people were recruited from the general community in the suburbs close to the Seniors Exercise Parks location in Melbourne, Australia. Advertisements in local newspapers, council newsletters, posters displayed on notice boards, and flyers distributed to senior groups were used for recruitment. Information was also placed online on the councils' and participating partners' websites as well as associated social media platforms (e.g. Facebook, Twitter). Recruitment took place between October 2018 to November 2019 . 


\section{Procedure}

Participants who met the inclusion criteria attended an initial (baseline) assessment at a community centre close to their area of residence. Demographic characteristics (age, gender), anthropometric measures (height and weight), previous medical history, current medication usage, socioeconomic and cultural background information (e.g. employment, level of education, country of birth, years of residency in Australia) and falls history were collected at baseline. Assessments were undertaken at baseline, 3 months and 9 months, by an allied health professional (Accredited Exercise Physiologist and/or Physiotherapist).

\section{Assessments}

\section{Primary outcome}

Physical activity The level of physical activity of the participants was measured using the Community Healthy Activities Model Program for Seniors (CHAM PS) [22]. The CHAMPS provides a self-reported measure of caloric expenditure (and frequency) per week in all exercise-related activities and caloric expenditure (and frequency) per week in moderate exercise -related activities.

\section{Secondary outcomes}

A comprehensive suite of physical function (strength, balance, functional mobility), psychosocial (quality of life, enjoyment, social isolation, fear of falls, loneliness), and mental health outcomes (mental wellbeing, depression) and falls risk assessment were undertaken as detailed in the protocol paper [19], and summarized below.

Physical function measures Physical measures of strength, balance and functional mobility were assessed using the following validated tests.

(i) Functional lower limb muscle strength was assessed using the 30-s sit to stand test [23]; (ii) Exercise tolerance and functional mobility was assessed using the two-minute walk test [24]; (iii) Dynamic balance was assessed using the step test [25], the sum of the number of steps from each limb was combined and used for the analysis [26]; and (iv) Walking speed was assessed using the $4 \mathrm{~m}$ walk test [27].

Psychosocial, mental and quality of life health outcomes Psychosocial, mental health and quality of life outcomes were assessed using the following questionnaires:

(i) Health-related quality of life was assessed using the EQ-5D-5L [28]. The EQ-5D-5L comprises five dimensions (mobility, self-care, usual activities, pain/ discomfort and anxiety/depression) as well as an overall self-rated health status (Visual Analog Scale
(VAS) 0-100) where higher score represents better health.

(ii) Mental wellbeing was assessed using the five-item World Health Organization (WHO-5) Wellbeing questionnaire $[29,30]$. The WHO-5 measures psychological wellbeing and depressive symptoms using 5 simple questions. The raw score was calculated to obtain a percentage score, which ranges from 0 representing the worst imaginable wellbeing and 100 representing the best imaginable well-being.

(iii) Loneliness was assessed using the UCLA 3-item Loneliness Scale which incorporates three dimensions of loneliness: relational connectedness, social connectedness and self-perceived isolation [31, 32]. The scale gives a possible range of scores from 3 to 9 (higher scores indicate greater feelings of loneliness).

(iv) Depression was assessed using the short version Geriatric Depression Scale (GDS-15) where a score of 0 to 5 is considered normal and a score greater than 5 suggests depressive symptoms [33].

(v) Fear of falls was assessed using The Short Falls Efficacy Scale International (Short FES-I) questionnaire [34], a 7-item scale ranging from 7 (no concern about falling) to a maximum 28 (severe concern about falling).

(vi) Self-efficacy barriers to exercise was assessed using The Self-Efficacy for Exercise (SEE) questionnaire, with scores ranging from 0 to 90 (a higher score indicates higher self-efficacy for exercise) [35].

(vii) Enjoyment was assessed using the 8-item version Physical Activity Enjoyment Scale (PACES), where higher values reflect greater levels of enjoyment (values range 8-56) [36].

(viii)Social isolation and social support were assessed using the short version 6-item Lubben Social Network Scale (LSNS6). The score ranges between 0 and 30 where higher scores indicate more social engagement [37].

\section{Falls risk assessment}

(i) The Falls Risk for Older People in the Community (FROP-Com) risk assessment tool was used to assess fall risk. The FROP-Com consists of 13 falls risk factor domains, with most risk factors scored to reflect graded risk on a 4-point scale (nil, mild, moderate, or severe) [38].

\section{Exercise park Intervention}

The Seniors Exercise Park

The Seniors Exercise Park equipment (Lark Industries (Australia) and Lappset Group) consists of outdoor playground equipment specifically designed for older people to improve strength, balance, joint movements and overall mobility and function (Fig. 2). It comprises multiple 
equipment stations that target a specific function or movement (upper and lower limb) such as shoulder range of movement, static and dynamic balance (unstable surfaces), and functional movements (walking up/down stairs, sit to stand). The exercise park equipment was installed in two public locations and a third location in a retirement living and aged care community respectively: Barry Rd. Community Centre, Thomastown, Melbourne (under the municipality of Whittlesea City Council); Central Park Community Centre, Hoppers Crossing, Melbourne (under the municipality of Wyndham City Council); and Leith Park, St Helena, Melbourne (Old Colonists' Association of Victoria).

\section{2-week structured supervised exercise program}

Participants participated in a 12-week supervised exercise intervention program twice a week using the Seniors Exercise Park. The exercise program was delivered by a qualified exercise instructor (Accredited Exercise Physiologist or Physiotherapist). Participants performed exercises that focused on strength, balance, coordination, mobility and flexibility as detailed in our previous work [39]. Each session consisted of 5-7 min of warm-up exercises, followed by $45-75 \mathrm{~min}$ at the equipment stations, and concluded with $5 \mathrm{~min}$ of cool down exercises (overall duration approximately $80 \mathrm{~min}$ ). The exercise classes were run as a circuit-based group program with 6-10 participants. Each participant was familiarized with the exercises individually and the difficulty level was tailored to the capabilities of the participant. Each session was followed by morning/ afternoon tea to encourage socialization.

Individual and group exercise progression Each exercise station included two different exercises which were performed twice by each participant. Examples of the stations and the exercises can be found at https://youtu.be/ PaYuCMtnlYk. Two participants were allocated to each station such that each participant performed one exercise for the allotted time and then swapped with their partner, repeating each exercise twice before rotating to the next station. Rest periods were provided during transition to the next station. The duration of each exercise and rest period were adjusted progressively according to program progression, as detailed in the protocol paper [19].

\section{Participation rate (adherence) and exercise monitoring}

During the 12-week supervised exercise program Frequency of exercise session participation was determined using daily attendance logs kept by the exercise instructor. Overall adherence to the structured exercise program was defined by the number of sessions attended: where $100 \%$ adherence indicated that a participant attended all available 24 sessions. In the event of cancellation (due to weather or public holidays), participants were given alternative make up sessions to achieve the 24 sessions.

Monitoring exercise uptake following the 12-week exercise program for $\mathbf{6}$ months - fob access system At the completion of the structured 12 weeks exercise program participants were given two options to choose from to continue their physical activity. Option 1 - independent unsupervised access and usage of the exercise park in participants' own preferred time. Option 2: access to twice a week exercise sessions on the exercise park under supervision but with no formal structured group activity. Adherence and exercise uptake for the 6 months post intervention was monitored using a fob access system (CityWatch Security, Melbourne, Australia www.citywatchsecurity.com.au/) that included a card reader/scanner and a control panel at each site. Participants were assigned an individual identification key (fob) which they used to tap a card reader each time they accessed the Seniors Exercise Park at the site. Their access was recorded and monitored (thereby electronically monitoring access). A separate paper will report outcomes and experiences using the fob system.

\section{Safety considerations and adverse events}

Weather elements In extreme weather conditions (e.g. heavy rain, extreme heat (above $30^{\circ} \mathrm{C}$ )), if deemed by the exercise instructor as unsafe to exercise, sessions were cancelled. In circumstances where sessions were cancelled, or during a holiday period, makeup sessions were organised towards the end of the program (up to two weeks or 4 sessions). Any cancellation and the associated reason were recorded in a log book kept by the researchers.

\section{Adverse events}

\section{Joint pain/discomfort and or muscle soreness}

Instances of joint pain or discomfort (directly related to the exercise program) during the exercise program were recorded. Sessions that were missed due to pain or discomfort that had not settled and prevented a participant from attending the exercise sessions were also recorded.

\section{Falls}

Any falls during the delivery of the structured supervised exercise programs and during the independent usage phase of the Seniors Exercise Park were recorded. A fall was defined as an event when the participant inadvertently comes to rest on the ground, floor or other lower level' (WHO Global Report on Falls Prevention in Older Age [40]).

Serious adverse events - cardiorespiratory adverse reaction Any report of difficulty breathing that did not settle quickly with rest, new or unrelenting chest pain, or acute changes in the level of consciousness during the session 
were documented. A serious adverse event was deemed if symptoms did not settle and medical emergency care was required and organised.

\section{Power analysis}

A power analysis was undertaken using previously published data using the primary outcome measure CHAMPS for measurement of change in physical activity level over a 9 month period [22]. We considered a minimum meaningful change in the physical activity outcome from use of the Seniors Exercise Park intervention to be $d=0.33$. Using this standardized effect size, $90 \%$ power and a twotailed alpha of 0.05 , we calculated need for a sample size of 98 participants. Previous data indicates a within subject change in daily calorie expenditure for all activities of 1509 and for moderate intensity activities of 1196 when exposed to 6-month physical activity program [22, 41]. This meant we were likely to have $90 \%$ power to detect a change of 503 in total daily calorie expenditure, and 399 in daily calorie expenditure in moderate intensity activities. We projected for a $15 \%$ drop-out rate, thus we sought a total sample of 113 participants (37-38 per site).

\section{Statistical analyses}

For the primary outcome of overall physical activity score (CHAMPS) and the physical, mental, and health secondary outcome measures, repeated measures analysis of variance (ANOVA) were used to determine if there were differences between scores collected at baseline assessment and at 9 month follow-up. A separate repeated measures ANOVA (with the equivalent non parametric test for ordinal data) was used to examine the effect of the exercise program on physical activity level, physical, mental and psychosocial and health outcomes between baseline and 3 months; and 3 months vs 9 months. Information collected about exercise adherence were reported using descriptive statistics (\% of adherence). Data were analysed using SPSS version 26.0 (IBM Corp, NY, USA). Effect size, Partial Eta Squared, ( $\eta_{p}^{2}$ ) from SPSS was used to determine effect size as follows: $\eta_{p}^{2}$ values greater than 0.14 were considered a large and significant effect size whereas 0.01 and 0.06 were considered small and medium, respectively [42].

\section{Results}

Ninety-five older people living in the community who volunteered to participate were eligible to take part in the study, with a mean age of $73.0 \pm 7.4$, and $82.1 \%$ female. The majority of participants (94.7\%) suffered from at least one medical condition with the most common conditions reported being arthritis (70.1\%), hypertension (62.1\%) and hypercholesterolemia (51.6\%) (Table 1). Fifteen participants dropped out between baseline and three months follow up (15.7\%), leaving 80 participants available for analysis of pre-post intervention (mean age $72.8 \pm 7.5$ years; $81.3 \%$ females). No significant differences existed between those who dropped out and the remaining sample with respect to their medical or demographic characteristics. Recruitment and drop out breakdown are provided in Fig. 1. Interruption to data collection occurred during the COVID-19 pandemic due to the physical distancing and lock down restrictions which prevented access to the Seniors Exercise Park. Participants were not able to access the Seniors Exercise Parks for a lengthy period of several months (restrictions of public parks closure and no access to aged care sites as imposed by the Australian State Government). Data of $n=19$ was impacted due to COVID-19 and were excluded from the analysis (Fig. 1). Consequently, a separate analysis was conducted for the comparison of the CHAMPS primary and secondary outcomes between baseline and 9 months follow up $(n=58)$, in addition to the baseline vs 3 months analyses $(n=80)$.

Average adherence in the supervised 12-week program was $86 \%$. The most frequently reported reason for absence from classes was due to illness or medical problems (37.6\% of the occasions of absences). Only $6.9 \%$ of sessions were cancelled due to weather (hot or wet). During the 12-week program, 12 people (15\%) reported pain or discomfort due to aggravation of pre-existing injury/condition, with 16 events $(0.95 \%$ of all sessions) reported. Five people (6.25\%) missed exercise sessions due to aggravation of pre-existing injury/condition with a total of 15 sessions missed ( $0.89 \%$ of all sessions). One fall occurred during the exercise program with no severe injury. No serious adverse events occurred during the program.

A significant increase in physical activity level was demonstrated following the intervention (CHAMPS caloric expenditure, frequency per week and total time in all exercise and in moderate exercise per week, $p<0.01$, moderate to large effect sizes). Significant improvements were also demonstrated in all physical function measures $(p<0.01$, small to large effect sizes), self rated quality of life $(p=0.04$, small effect size), wellbeing $(p<0.01$, small effect size), fear of falls ( $p<0.01$, medium effect size), falls risk ( $p<0.01$, medium effect size), depressive symptoms ( $p=0.01$, small effect size) and loneliness $(p=0.03$, small effect size). No significant changes were demonstrated in socialisation and self-efficacy for exercise outcomes $(p>0.05)$ (Table 2). Changes in the EQ-5D-5L dimensions are presented in Fig. 3 with improvements shown in selfcare $(p<0.01)$ and depression $(p=0.02)$ domains.

For the 9 month follow up $(n=58)$ significant improvements from baseline were demonstrated in the frequency, duration and caloric expenditure of moderate physical activity and all type of exercises $(p<0.05$, moderate to large effects size, Table 2). Significant improvements in all physical function measures were demonstrated between 
Table 1 Participants' characteristics

\begin{tabular}{|c|c|c|c|}
\hline & $\begin{array}{l}\text { Overall sample } \\
n=95\end{array}$ & $\begin{array}{l}\text { Completed intervention } \\
n=80\end{array}$ & $\begin{array}{l}\text { Drop out } \\
n=15\end{array}$ \\
\hline Age (yrs), mean $\pm S D$ & $73.0 \pm 7.4$ & $72.8 \pm 7.5$ & $74.13 \pm 7.03$ \\
\hline Females (\%) & $78(82.1)$ & $65(81.3)$ & $13(86.7)$ \\
\hline Height $(m)$, mean $\pm S D$ & $1.6 \pm 0.08$ & $1.6 \pm 0.9$ & $1.6 \pm 0.7$ \\
\hline Weight $(\mathrm{kg})$, mean $\pm \mathrm{SD}$ & $78.3 \pm 16.8$ & $76.6 \pm 15.2$ & $87.1 \pm 22.1$ \\
\hline $\mathrm{BMI}\left(\mathrm{kg} / \mathrm{m}^{2}\right)$, mean $\pm \mathrm{SD}$ & $29.9 \pm 5.8$ & $29.3 \pm 5.4$ & $32.9 \pm 7.3$ \\
\hline Falls in preceding 12 months (\%) & $49(51.5 \%)$ & $42(52.5 \%)$ & $7(46.6)$ \\
\hline \multicolumn{4}{|l|}{ Medical conditions and musculoskeletal conditions n (\%) } \\
\hline Arthritis (Osteoarthritis/Rheumatoid Arthritis) & $67(70.5)$ & $55(68)$ & $12(80)$ \\
\hline Hypertension & $59(62.1)$ & $48(60)$ & $11(73.3)$ \\
\hline Hypercholesterolemia & 49 (51.6) & $44(55)$ & $5(33.3)$ \\
\hline Hearing impairments & $43(45.3)$ & $31(38.7)$ & $12(80)$ \\
\hline Cardiovascular conditions & $28(29.5)$ & $22(27.5)$ & $6(40)$ \\
\hline Incontinence & $26(27.4)$ & $20(25)$ & $6(40)$ \\
\hline Respiratory conditions & $24(25.3)$ & $18(22.5)$ & $6(40)$ \\
\hline Osteoporosis & $21(22.1)$ & $19(23.8)$ & $2(13.3)$ \\
\hline Diabetes mellitus & $18(18.9)$ & $15(18.8)$ & $3(20)$ \\
\hline Other metabolic conditions (Kidney/Thyroid Disorder) & $28(29.4)$ & $20(25)$ & $8(53.3)$ \\
\hline \multicolumn{4}{|l|}{ Medication usage and type, n (\%) } \\
\hline Taking medications & $85(89.4)$ & $71(90)$ & $14(93.3)$ \\
\hline Median number of medications (Interquartile Range) & $6(5)$ & $6(6)$ & $6(4)$ \\
\hline Hypertensive medications & $59(62.1)$ & $47(58.8)$ & $12(80)$ \\
\hline Cholesterol-lowering medications & $45(47.3)$ & $40(50)$ & $5(33.3)$ \\
\hline Blood Thinners & $29(30.5)$ & $25(31.3)$ & $4(26.7)$ \\
\hline Pain relieving medications & $26(27.4)$ & $26(32.5)$ & $2(13.3)$ \\
\hline Anti-depressant medications & $21(22.1)$ & $16(20)$ & $5(33.3)$ \\
\hline Respiratory medications & $19(20)$ & $16(20)$ & $3(20)$ \\
\hline Glucose lowering medications & $13(13.7)$ & $12(15)$ & $2(13.3)$ \\
\hline Anti-inflammatory medications & $10(10.5)$ & $9(11.3)$ & $1(6.7)$ \\
\hline \multicolumn{4}{|l|}{ Socio-economic and education status } \\
\hline Education level - Secondary school or below (\%) & $60(63.1)$ & $50(62.5)$ & $10(66)$ \\
\hline First Generation migrants (born overseas) (\%) & $\begin{array}{l}35 \text { (36.8) } \\
\text { Europe } 20 \text { (57.1); Middle East } 6 \\
\text { (17.1); Asia } 5 \text { (14.3); America } 1 \\
\text { (2.8); South Africa } 2 \text { (5.7), } \\
\text { Oceania } 1 \text { (2.8) }\end{array}$ & $\begin{array}{l}32(40) \\
\text { Europe } 17 \text { (53.1); Middle East } 6 \\
\text { (18.8); Asia } 5 \text { (15.6); America } 2 \\
\text { (3.1); South Africa } 1 \text { (6.3), } \\
\text { Oceania } 1 \text { (3.1) }\end{array}$ & $\begin{array}{l}3(20) \\
\text { Europe } 3(100)\end{array}$ \\
\hline Second Generation migrants (parents born overseas) & $51(53.6)$ & $44(55)$ & $7(46.7)$ \\
\hline Speak other language than English & $18(18.9)$ & $16(20)$ & $2(13.3)$ \\
\hline \multicolumn{4}{|l|}{ Marital status } \\
\hline Married/spouse & $50(52.6)$ & $43(53.8)$ & $7(46.7)$ \\
\hline Widowed & $22(23.2)$ & $17(21.2)$ & $5(33.3)$ \\
\hline Single/divorced/separated & $23(24.2)$ & $20(25)$ & $3(20)$ \\
\hline
\end{tabular}




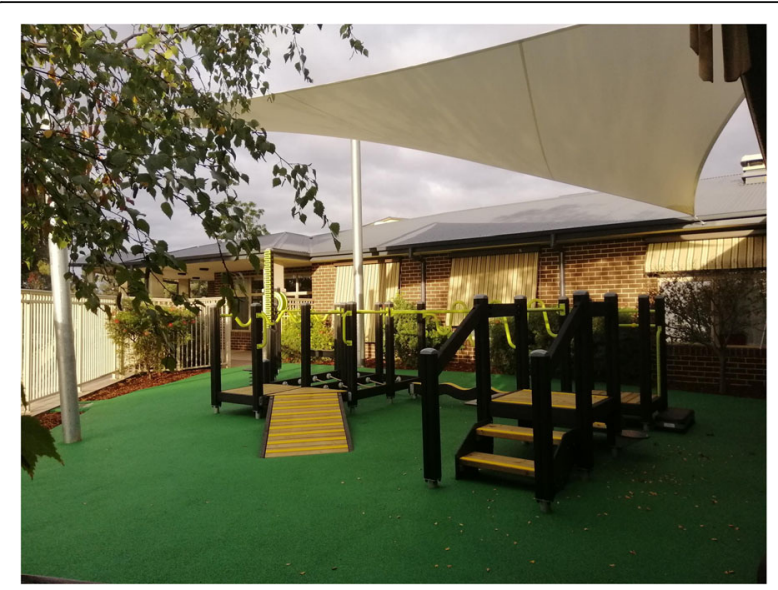

Fig. 2 The Seniors Exercise Park at Leith Park, St Helena

baseline and 9 months follow up $(p<0.05$, moderate to large effect size, Table 2). Significant changes were observed only in the health related quality of life mobility and self-care domains $(p<0.05$, EQ-5D-5L dimensions, Fig. 3). No changes were observed in the other health related quality of life measures apart from significant reductions in both fear of falls and falls risk $(p<0.01$, Table 2$)$. Very few changes were observed between 3 and 9 month follow ups as indicated in Table 2.

\section{Discussion}

Participation in physical activity is important for the reduction and management of chronic diseases as well as to help older people remain mobile and independent. Only a quarter of older people meet the recommended national guidelines for physical activity [43]. Participation in the ENJOY Seniors Exercise Park program resulted in increases in physical activity level as well as physical and mental health benefits in the short term with sustained physical function benefit in the longer term.

Although there is strong evidence from randomized controlled trials to support the importance of physical activity, older people have low participation rates in physical activity programs aimed at achieving a variety of positive health outcomes, including falls prevention $[44,45]$. Those who do commence a physical activity program as part of a research study often return to their inactive lifestyle behaviour once the study is completed, indicating that interventions that are not easy to apply in "real world" situations often do not sustain participation beyond the trial period [9]. Hence translating these studies into effective and sustained behaviour change remains a challenge [46]. Participation in the ENJOY project resulted in significant improvement in physical activity level (all CHAMPS outcomes) after the 12 weeks intervention and these improvements were sustained 6 months later (with no decline between 3 and 9 months), suggesting that participants remained physically active over the longer term. Importantly, the increase in the moderate exercise type (frequency and duration) indicates that participants exceeded the recommended physical activity participation of $150 \mathrm{~min}$ per week of moderate intensity. The sustained engagement in physical activity provides promising results for the potential benefits of scaling up such a program in order to achieve public health benefit for older people in the community.

Sustained participation in physical activity at a level to maintain or improve health by older people remains challenging to achieve, with fewer than half of older adults being active enough to achieve most of these health benefits [7]. Having national and international guidelines or recommendations appear insufficient to achieve this activity [47]. Providing widely available and accessible avenues for physical activity options, such as widespread implementation of outdoors Seniors Exercise Parks may assist improving participation levels. State and / or national policies supporting increased physical activity participation by older people may also be beneficial.

Loneliness and social isolation are greatest among older people and can pose significant physical and mental health risks $[48,49]$. Loneliness, in addition to other physical and mental problems, gives rise to feelings of depression in older people [50]. Physical activity is one possible health promotion strategy that has positive effects on mental health in later life [51]. Improvement in depressive symptoms (as reported by the Depression domain in the Quality of Life scale and the Geriatric Depression Scale) and wellbeing were seen after the 3 months intervention. Slight improvement in loneliness (relational connectedness, social connectedness and self-perceived isolation) was demonstrated but with no changes in social isolation or social support. Caution must be taken, however, in the interpretation of the results as the values reported in both scales (UCLA3 and LSNS6) did not suggest that the participants experienced severe loneliness or lack of social engagement at baseline. The physical activity program incorporated group setting exercise activity followed by a social morning tea, which facilitated social connection. Enjoyment in physical activity was also significantly improved following the intervention. Given that fun, enjoyment and social interaction are key motivators for older people to take part in physical activity [52], these aspects should be an integral part of physical activity programs for older people. Consequently, the results highlight the beneficial effect of the Seniors Exercise Park program on general wellbeing.

We have previously demonstrated in a small randomized controlled trial the physical and social benefits of utilizing the Seniors Exercise Park program for older people [15, 16]. Pre-post studies such as the ENJOY trial may be considered to be less rigorous than randomised controlled trials, although in implementation research, especially where 


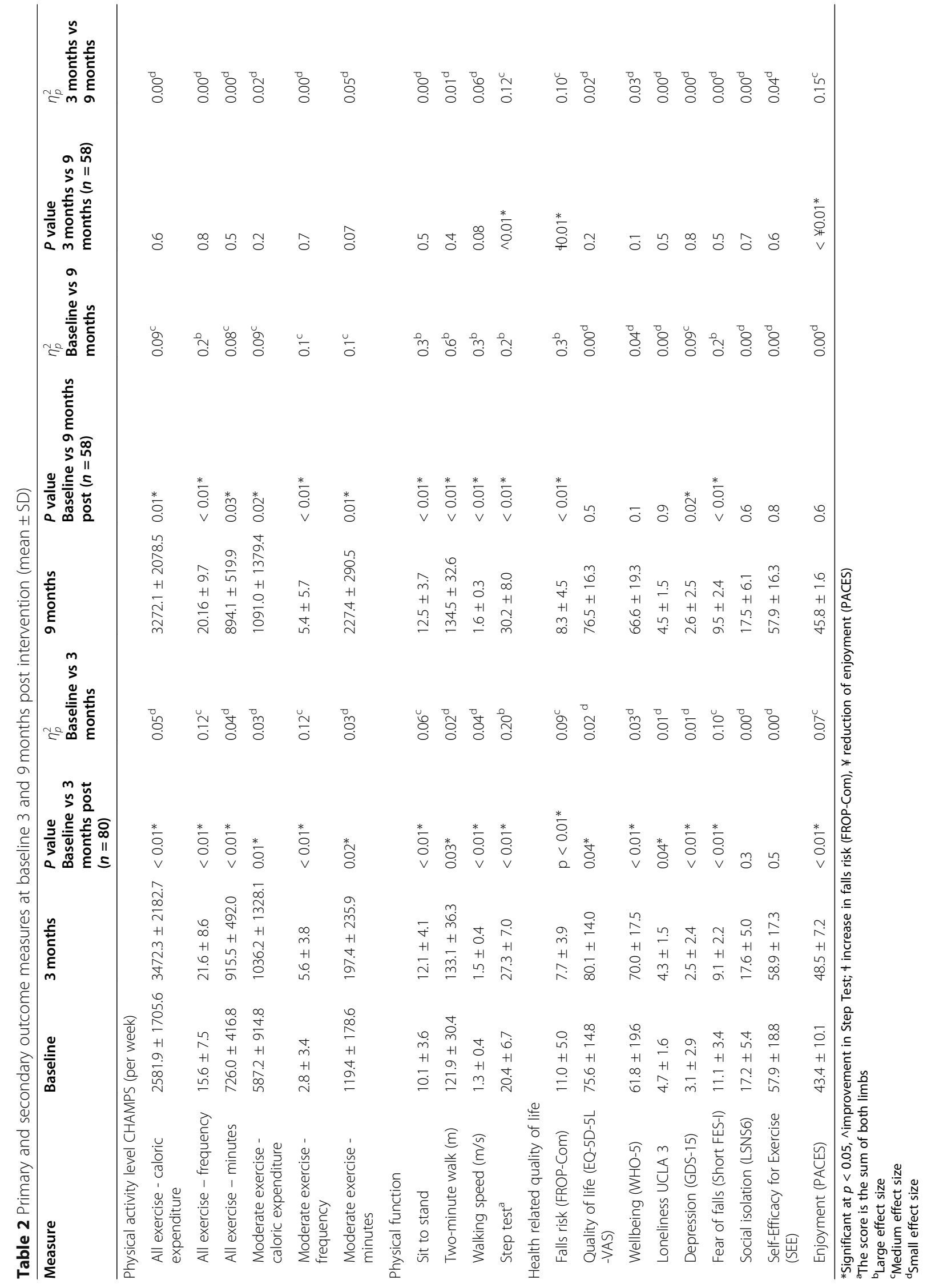




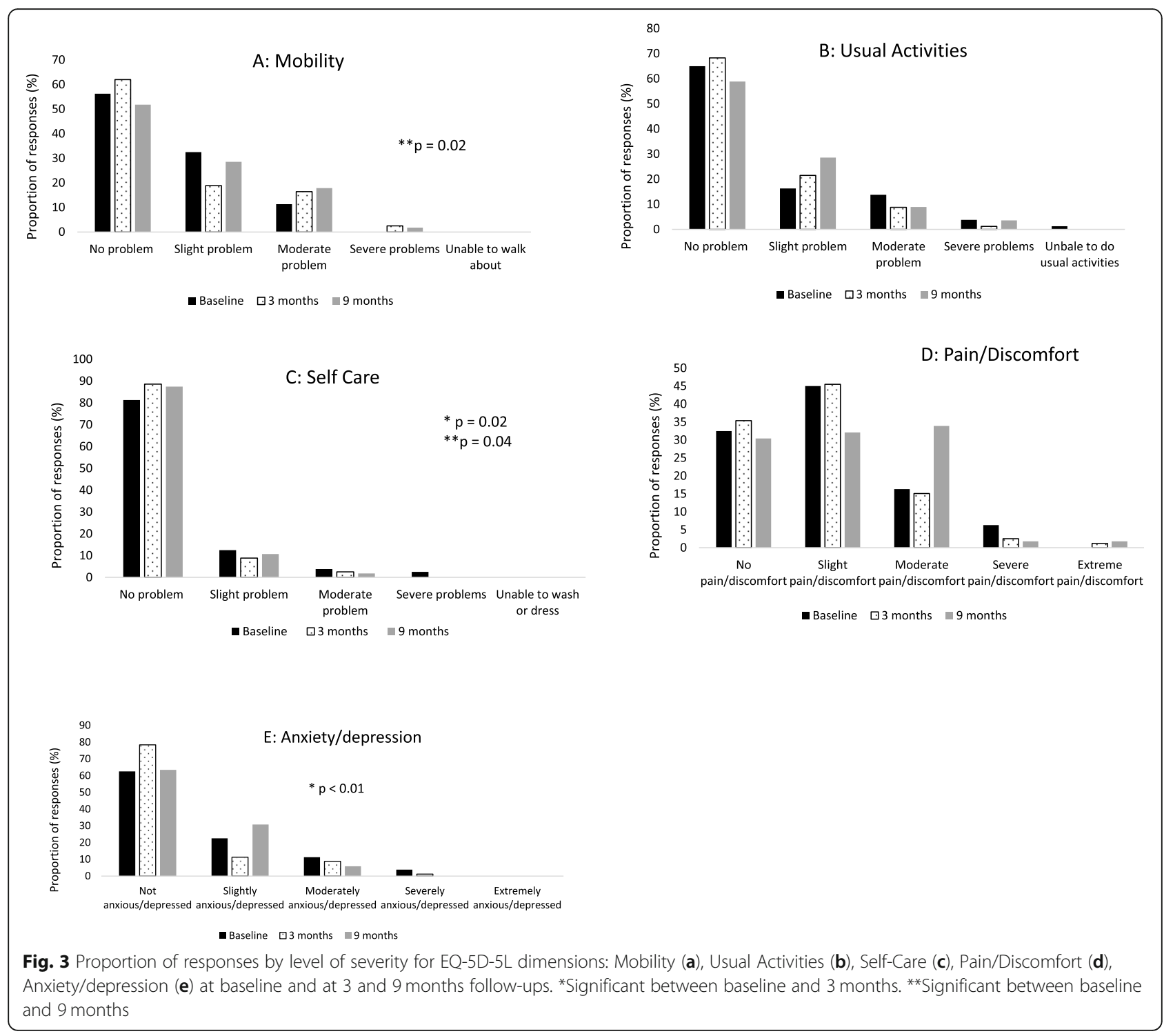

one or more previous randomised trials have demonstrated the approach to be effective (as in this study), this is considered acceptable [53]. The ENJOY project provides further evidence for the potential effectiveness of the physical and social activity program to improve quality of life and wellbeing on a larger scale beyond the 12 weeks supervised program. The approach utilized in the ENJOY project encompasses partnership with local governments to create an innovative enjoyable physical activity for older people with the utilization of specialized outdoor equipment. The availability of the equipment in community settings provides an advantageous set up where participants can have free access beyond the research trial. Moreover, the location in outdoor settings also offers additional benefits, as exercising outdoors is known for its' health benefits for mental wellbeing [54]. Combining exercise, nature and social components may play a key role in engaging older people in physical activity and health promotion initiatives longer term.

Older people are at high risk of falls. Exercise programs have been shown to be effective in preventing falls in community-dwelling older people [55]. Balance and strengthening exercises in particular are important to be incorporated into exercise programs to reduce falls [55]. The Seniors Exercise Park program integrates multimodal exercise stations that target balance (unstable/uneven surfaces), strength and functional movements. This offers an important combination of different physiological aspects to obtain broader health benefits in addition to falls prevention, and is different to what is available in most traditional outdoor exercise equipment, which is usually focused on either cardiorespiratory or strength training [56]. The current sample population included people with high risk of falls (52.5\% had previous falls) as well as high 
prevalence of complex medical issues. The sustained improvements in the physical function measures as well as the reduction in fear of falls and falls risk at 9 months suggest potential benefits to reduce the risk of falls.

This study has several limitations. Firstly, the spread of COVID-19 in early 2020 and associated restrictions and lockdown prevented older people in the community to be physically active using the Seniors Exercise Park in the latter stages of this project. This has adversely impacted on the ENJOY project data collection as participants were unable to access the Seniors Exercise Park sites as well as their ability to maintain their physical activity using this exercise modality. As such the data for the 9 months follow up included a smaller sample which could have potentially impacted on the results, leading to underestimation of the impact of the physical activity program on various health measures. The total dropout rate ( $n=18$, Fig. 1$)$ of those who ceased participation in the project was $18.9 \%$ which is within the expected rate for exercise related interventional studies among older people $[57,58]$. Secondly, the level of physical activity was measured using self-reported questionnaires which might not accurately reflect the actual physical activity level of the participants. However it is important to acknowledge that the CHAMPS questionnaire has been widely used in research and has been designed for use in evaluating interventions that primarily aim to increase levels of physical activity in older adults [22]. It is a reliable and valid questionnaire that is sensitive to change of the physical activity measures derived from it. The questionnaire was also tested and found suitable to be used with older Australians [59]. Finally, while our sample is representative of older Australians' age, BMI and cultural background, it included a relatively high proportion of females with a small proportion of male participants. Although the risk and incidence of falls are greater in females, males are commonly underrepresented in exercise intervention trials $[60,61]$. Males have been reported to have specific preferences and characteristics of exercise interventions that are most likely to appeal to them [62]. The specific appeal or lack of appeal of this outdoor exercise park approach will need to be explored in future research.

\section{Conclusion}

The results suggest that the Seniors Exercise Park may be an effective modality for improving older people's physical function and wellbeing beyond an initial supervised program, and can be an important public health infrastructure investment in promoting physical activity for older people. Future work should focus on wider implementation of the program and on scaling up this initiative to achieve public health benefit for the community.

\section{Abbreviations}

ENJOY: Exercise interveNtion outdoor proJect in the cOmmunitY for older people; RCT: Randomized controlled trial; TREND: Transparent Reporting of Evaluations with Nonrandomized Designs; CONSORT: Consolidated Standards Of Reporting Trials; AMTS: Abbreviated Mental Test Score; CHAMPS: Community Healthy Activities Model Program for Seniors; EQ-5D-5L: The 5-level EQ-5D; UCLA: University of California at Los Angeles Loneliness Scale; GDS-15: Geriatric Depression Scale; Short FES-I: Short Falls Efficacy Scale International; SEE: SelfEfficacy for Exercise; PACES: Physical Activity Enjoyment Scale; FROP-Com: The Falls Risk for Older People in the Community; SD: Standard deviation; LSNS6: 6 items Lubben Social Network Scale; WHO-5: World Health Organization Five wellbeing index

\section{Acknowledgments}

The authors would like to acknowledge Whittlesea City Council, Wyndham City Council and Old Colonists' Association of Victoria for their collaboration and partnership in this project. Moreover, we would like to thank Lark Industries for the Seniors Exercise Park equipment installation and associated advice and support.

\section{Authors' contributions}

$\mathrm{PL}, \mathrm{KH}, \mathrm{TH}, \mathrm{SB}, \mathrm{GD}, \mathrm{BD}$ participated in the design and conception of the project. $\mathrm{PL}, \mathrm{MP}, \mathrm{JD}, \mathrm{FB}, \mathrm{KH}, \mathrm{BD}$ were involved in the project management planning and in community organisation and local governments participation. PL, MP, JD conducted the intervention and collected the data. $P L, K H, T H$ led the statistical analysis plan. PL, MP, JD, TH, FB, BD, SB, GD, KH participated in data interpretation. PL, KH drafted the manuscript. MP, JD, TH, $\mathrm{FB}, \mathrm{BD}, \mathrm{SB}, \mathrm{GD}$ read and revised the manuscript. All authors have read and approved the final version.

\section{Funding}

This study was funded by Gandel Philanthropy. This funding source had no role in the design of the study, its execution, analyses, interpretation of the data, and in writing the manuscript for publication.

\section{Availability of data and materials \\ The datasets generated and/or analysed during the current study are not publicly available due to ethical restrictions but are available from the corresponding author on reasonable request.}

\section{Ethics approval and consent to participate}

Ethical approval has been obtained from the Melbourne Health Human Research Ethics Committee, Melbourne (Application ID. HREC/18/MH/286, local number 2018.238). All participants signed a consent form prior to participation.

\section{Consent for publication \\ Not Applicable.}

\section{Competing interests}

The authors declare that they have no competing interests.

\begin{abstract}
Author details
${ }^{1}$ National Ageing Research Institute, Melbourne, Australia. ${ }^{2}$ Institute for Health and Sport, Victoria University, Melbourne, Australia. ${ }^{3}$ Rehabilitation, Ageing and Independent Living Research Centre, Monash University, Melbourne, Australia. ${ }^{4}$ School of Primary and Allied Health Care, Monash University, Melbourne, Australia. ${ }^{5}$ Centre for Health Policy, University of Melbourne, Melbourne, Australia. ${ }^{6}$ School of Nursing and Midwifery, Deakin University, Melbourne, Australia. ${ }^{7}$ Department of Physiotherapy, The University of Melbourne, Melbourne, Australia. ${ }^{8}$ Centre for Health Research, University of Southern Queensland, Brisbane, Australia. ${ }^{9}$ Australian Institute for Musculoskeletal Science (AIMSS), Melbourne Medical School, Melbourne University, Melbourne, Australia.
\end{abstract}

Received: 1 July 2020 Accepted: 7 October 2020

Published online: 04 November 2020

\section{References}

1. World Population Ageing 2019: Highlights. In. New York: United Nations, Department of Economic and Social Affairs, Population Division; 2019. 
2. Cooper C, Hagan P. The ageing Australian population and future health costs: 1996-2051. Department of Health and Aged Care Occasional Papers: New Series No. 7;1999.

3. Blondell SJ, Hammersley-Mather R, Veerman JL. Does physical activity prevent cognitive decline and dementia?: a systematic review and metaanalysis of longitudinal studies. BMC Public Health. 2014;14:510.

4. Pratt M, Norris J, Lobelo F, Roux L, Wang G. The cost of physical inactivity: moving into the 21st century. Br J Sports Med. 2014;48(3):171-3.

5. Hallal PC, Andersen LB, Bull FC, Guthold R, Haskell W, Ekelund U. Global physical activity levels: surveillance progress, pitfalls, and prospects. Lancet. 2012;380(9838):247-57.

6. Australian Bureau of Statistics. Physical activity in Australia: a snapshot, 2007-08 In. Canberra; 2011.

7. Sun F, Norman IJ, While AE. Physical activity in older people: a systematic review. BMC Public Health. 2013:13:449.

8. Sansano-Nadal O, Gine-Garriga M, Brach JS, Wert DM, Jerez-Roig J, Guerra-Balic M, Oviedo G, Fortuno J, Gomara-Toldra N, Soto-Bagaria L, et al. Exercise-based interventions to enhance long-term sustainability of physical activity in older adults: a systematic review and metaanalysis of randomized clinical trials. Int J Environ Res Public Health. 2019;16(14):2527.

9. Li F, Eckstrom E, Harmer P, Fitzgerald K, Voit J, Cameron KA. Exercise and fall prevention: narrowing the research-to-practice gap and enhancing integration of clinical and community practice. J Am Geriatr Soc. 2016;64(2):425-31.

10. Kahn EB, Ramsey LT, Brownson RC, Heath GW, Howze EH, Powell KE, Stone EJ, Rajab MW, Corso P. The effectiveness of interventions to increase physical activity. A systematic review. Am J Prev Med. 2002;22(4 Suppl):73-107.

11. Bock C, Jarczok MN, Litaker D. Community-based efforts to promote physical activity: a systematic review of interventions considering mode of delivery, study quality and population subgroups. J Sci Med Sport. 2014;17(3):276-82.

12. Cohen DA, Marsh T, Williamson S, Golinelli D, McKenzie TL. Impact and costeffectiveness of family fitness zones: a natural experiment in urban public parks. Health Place. 2012;18(1):39-45.

13. Lee JLC, Lo TLT, Ho RTH. Understanding outdoor gyms in public open spaces: a systematic review and integrative synthesis of qualitative and quantitative evidence. Int J Environ Res Public Health. 2018;15(4):590.

14. WHO. Global action plan on physical activity 2018-2030: more active people for a healthier world. Geneva: World Health Organization; 2018.

15. Sales M, Polman R, Hill K, Levinger P. Older adults' perceptions to a novel outdoor exercise initiative: a qualitative analysis. J Aging Soc Change. 2018; 8(1):61-78.

16. Sales M, Polman R, Hill KD, Levinger P. A novel exercise initiative for seniors to improve balance and physical function. J Aging Health. 2017;29(8):1424.

17. Des Jarlais DC, Lyles C, Crepaz N, Group T. Improving the reporting quality of nonrandomized evaluations of behavioral and public health interventions: the TREND statement. Am J Public Health. 2004;94(3): 361-6.

18. Schulz KF, Altman DG, Moher D. CONSORT 2010 statement: updated guidelines for reporting parallel group randomised trials. BMC Med. 2010;8(1):18.

19. Levinger P, Panisset M, Dunn J, Haines T, Dow B, Batchelor F, Biddle S, Duque $\mathrm{G}$, Hill KD. Exercise interveNtion outdoor proJect in the cOmmunitY for older people - the ENJOY Senior Exercise Park project translation research protocol. BMC Public Health. 2019;19(1):933.

20. Hodkinson HM. Evaluation of a mental test score for assessment of mental impairment in the elderly. Age Ageing. 1972;1(4):233-8.

21. Sims J, Hill K, Hunt S, Haralambous B. Physical activity recommendations for older Australians. Australas J Ageing. 2010;29(2):81-7.

22. Stewart AL, Mills KM, King AC, Haskell WL, Gillis D, Ritter PL. CHAMPS physical activity questionnaire for older adults: outcomes for interventions. Med Sci Sports Exerc. 2001;33(7):1126-41.

23. Jones CJ, Rikli RE, Beam WC. A 30-s chair-stand test as a measure of lower body strength in community-residing older adults. Res Q Exerc Sport. 1999;70(2):113-9.

24. Butland RJ, Pang J, Gross ER, Woodcock AA, Geddes DM. Two-, six-, and 12minute walking tests in respiratory disease. Br Med J (Clin Res Ed). 1982; 284(6329):1607-8

25. Hill K, Bernhardt J, McGann A, Maltese D, Berkovits D. A new test of dynamic standing balance for stroke patients: reliability, validity and comparison with health elderly. Physiother Can. 1996;48:257-62.

26. Mercer VS, Freburger JK, Chang SH, Purser JL. Step test scores are related to measures of activity and participation in the first 6 months after stroke. Phys Ther. 2009;89(10):1061-71.
27. Studenski S, Perera S, Patel K, Rosano C, Faulkner K, Inzitari M, Brach J, Chandler J, Cawthon P, Connor EB, et al. Gait speed and survival in older adults. JAMA. 2011;305(1):50-8.

28. Greiner W, Weijnen T, Nieuwenhuizen M, Oppe S, Badia X, Busschbach J, Buxton M, Dolan P, Kind P, Krabbe P, et al. A single European currency for EQ-5D health states. Results from a six-country study. Eur J Health Econ. 2003;4(3):222-31.

29. Bonsignore M, Barkow K, Jessen F, Heun R. Validity of the five-item WHO well-being index (WHO-5) in an elderly population. Eur Arch Psychiatry Clin Neurosci. 2001;251(Suppl 2):II27-31.

30. WHO. Regional Office for Europe (1998) wellbeing measures in primary health care: The DepCare Project. In: Consensus meeting. Stockholm: World Health Organization; 1998.

31. Russell D, Peplau LA, Ferguson ML. Developing a measure of loneliness. J Pers Assess. 1978;42(3):290-4.

32. Russell DW. UCLA loneliness scale (version 3): reliability, validity, and factor structure. J Pers Assess. 1996;66(1):20-40.

33. Sheikh Jl, Yesavage JA. Geriatric depression scale (GDS) Recent evidence and development of a shorter version. In: TL B, editor. Clinical gerontology : a guide to assessment and intervention. New York: The Haworth Press; 1986. p. 165-73.

34. Kempen Gl, Yardley L, van Haastregt JC, Zijlstra GA, Beyer N, Hauer K, Todd C. The short FES-l: a shortened version of the falls efficacy scale-international to assess fear of falling. Age Ageing. 2008;37(1):45-50.

35. Resnick B, Jenkins LS. Testing the reliability and validity of the self-efficacy for exercise scale. Nurs Res. 2000;49(3):154-9.

36. Mullen SP, Olson EA, Phillips SM, Szabo AN, Wojcicki TR, Mailey EL, Gothe NP, Fanning JT, Kramer AF, McAuley E. Measuring enjoyment of physical activity in older adults: invariance of the physical activity enjoyment scale (paces) across groups and time. Int J Behav Nutr Phys Act. 2011:8:103

37. Lubben J, Blozik E, Gillmann G, lliffe S, von Renteln KW, Beck JC, Stuck AE. Performance of an abbreviated version of the Lubben social network scale among three European community-dwelling older adult populations. The Gerontologist. 2006;46(4):503-13.

38. Russell MA, Hill KD, Blackberry I, Day LM, Dharmage SC. The reliability and predictive accuracy of the falls risk for older people in the community assessment (FROP-com) tool. Age Ageing. 2008;37(6):634-9.

39. Sales MP, Polman R, Hill KD, Karaharju-Huisman T, Levinger P. A novel dynamic exercise initiative for older people to improve health and well-being: study protocol for a randomised controlled trial. BMC Geriatr. 2015;15:68.

40. WHO global report on falls prevention in older age. Geneva: World Health Organisation; 2007.

41. Stewart AL, Verboncoeur CJ, McLellan BY, Gillis DE, Rush S, Mills KM, King AC, Ritter P, Brown BW Jr, Bortz WM 2nd. Physical activity outcomes of CHAMPS II: a physical activity promotion program for older adults. $J$ Gerontol A Biol Sci Med Sci. 2001;56(8):M465-70.

42. Cohen J. Statistical power analysis for the behavioral sciences. 2nd ed. Hillsdale: Lawrence Erlbaum Assiciates; 1988

43. AlHW. In: 2018. AloHaW, editor. Physical activity across the life stages. Canberra: AlHW; 2018.

44. Nyman SR, Victor CR. Older people's participation in and engagement with falls prevention interventions in community settings: an augment to the cochrane systematic review. Age Ageing. 2012;41(1):16-23.

45. van der Deijl M, Etman A, Kamphuis CB, van Lenthe FJ. Participation levels of physical activity programs for community-dwelling older adults: a systematic review. BMC Public Health. 2014;14:1301.

46. Child S, Goodwin V, Garside R, Jones-Hughes T, Boddy K, Stein K. Factors influencing the implementation of fall-prevention programmes: a systematic review and synthesis of qualitative studies. Implement Sci. 2012;7:91.

47. WHO. Global rocommendations on physical activity for health. Geneva: World Health Organisation; 2011. https://www.who.int/dietphysicalactivity/ factsheet_recommendations/en/.

48. Beller J, Wagner A. Loneliness, social isolation, their synergistic interaction, and mortality. Health Psychol. 2018;37(9):808-13.

49. Ong AD, Uchino BN, Wethington E. Loneliness and health in older adults: a mini-review and synthesis. Gerontology. 2016;62(4):443-9.

50. Singh A, Misra N. Loneliness, depression and sociability in old age. Ind Psychiatry J. 2009;18(1):51-5.

51. Lautenschlager NT, Almeida OP, Flicker L, Janca A. Can physical activity improve the mental health of older adults? Ann Gen Hosp Psychiatry. 2004;3(1):12. 
52. Devereux-Fitzgerald A, Powell R, Dewhurst A, French DP. The acceptability of physical activity interventions to older adults: a systematic review and meta-synthesis. Soc Sci Med. 2016;158:14-23.

53. Curran GM, Bauer M, Mittman B, Pyne JM, Stetler C. Effectivenessimplementation hybrid designs: combining elements of clinical effectiveness and implementation research to enhance public health impact. Med Care. 2012;50(3):217-26.

54. Barton J, Griffin M, Pretty J. Exercise-, nature- and socially interactive-based initiatives improve mood and self-esteem in the clinical population. Perspect Public Health. 2012;132(2):89-96.

55. Sherrington C, Fairhall N, Wallbank G, Tiedemann A, Michaleff ZA, Howard K, Clemson L, Hopewell S, Lamb S. Exercise for preventing falls in older people living in the community: an abridged Cochrane systematic review. Br J Sports Med. 2019;54(15):885-91.

56. Levinger P, Panisset M, Parker H, Batchelor F, Tye M, Hill KD. Guidance about age-friendly outdoor exercise equipment and associated strategies to maximise usability for older people. Health Promot J Austr. 2020. p. 1-8.

57. Picorelli AM, Pereira LS, Pereira DS, Felicio D, Sherrington C. Adherence to exercise programs for older people is influenced by program characteristics and personal factors: a systematic review. J Physiother. 2014;60(3):151-6.

58. Jancey J, Lee A, Howat P, Clarke A, Wang K, Shilton T. Reducing attrition in physical activity programs for older adults. J Aging Phys Act. 2007;15(2):152-65.

59. Cyarto EV, Marshall AL, Dickinson RK, Brown WJ. Measurement properties of the CHAMPS physical activity questionnaire in a sample of older Australians. J Sci Med Sport. 2006;9(4):319-26.

60. Waters LA, Galichet B, Owen N, Eakin E. Who participates in physical activity intervention trials? J Phys Act Health. 2011;8(1):85-103.

61. Ng C, Fairhall N, Wallbank G, Tiedemann A, Michaleff ZA, Sherrington C. Exercise for falls prevention in community-dwelling older adults: trial and participant characteristics, interventions and bias in clinical trials from a systematic review. BMJ Open Sport Exerc Med. 2019;5(1):e000663.

62. Chatfield SL, Gamble A, Hallam JS. Men's preferences for physical activity interventions: an exploratory study using a factorial survey design created with R software. Am J Mens Health. 2018;12(2):347-58.

\section{Publisher's Note}

Springer Nature remains neutral with regard to jurisdictional claims in published maps and institutional affiliations.

Ready to submit your research? Choose BMC and benefit from:

- fast, convenient online submission

- thorough peer review by experienced researchers in your field

- rapid publication on acceptance

- support for research data, including large and complex data types

- gold Open Access which fosters wider collaboration and increased citations

- maximum visibility for your research: over $100 \mathrm{M}$ website views per year

At $\mathrm{BMC}$, research is always in progress.

Learn more biomedcentral.com/submissions 